\title{
Another Technique for Contrast-Free Deep Cannulation of Common Bile Duct
}

In patients with obstructive jaundice, if the contrast is injected without securing deep cannulation in the bile duct the endoscopic drainage procedure may fail, necessitating urgent surgery or percutaneous drainage to avoid infection; death may ensue. We describe our method of deep cannulation of the bile duct prior to injection of contrast.

For selective deep cannulation of the common bile duct we load the ERCP catheter with sterile normal saline to prevent entry of air bubbles into the bile duct. A 0.035 inch guidewire is passed through the catheter and this assembly is introduced into the biopsy channel of a side-viewing therapeutic endoscope. The ERCP catheter is introduced into the punctum, in the direction of the bile duct. Under fluoroscopic guidance the guidewire is gently advanced into the bile duct and then pushed deep into it (Figures 1 and 2).

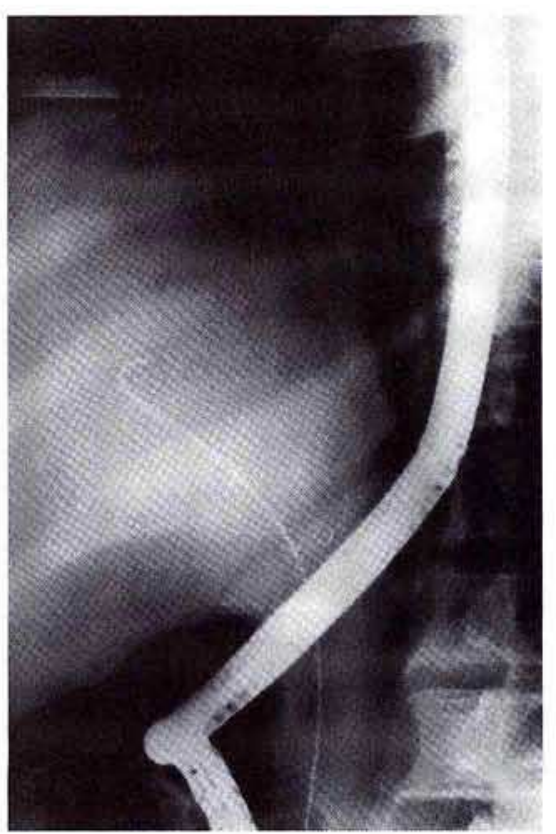

The cannula is then threaded over the guidewire. Once the guidewire and cannula assembly has reached the level of confluence of the hepatic ducts or the intrahepatic biliary radicles, the guidewire is withdrawn and contrast-free bile is aspirated for culture and analysis; contrast is then injected for the cholangiogram. After performing the cholangiogram the guidewire is reintroduced and different therapeutic procedures, such as wire-guided sphincterotomy, nasobiliary drainage or biliary stenting, are performed.

After securing deep access in the bile duct the contrast is injected so that there is no chance of the drainage procedures failing; bile is drained before injecting the contrast, which is preferred in patients with suppurative cholangitis to prevent septicaemia $(1,2)$. In the past $3 \mathrm{~F}$ biliary catheters were used to achieve deep cannulation of the bile duct to harvest contrast-free bile for

Figures 1,2: X-ray pictures showing guidewire deep in region of intrahepatic bile ducts.

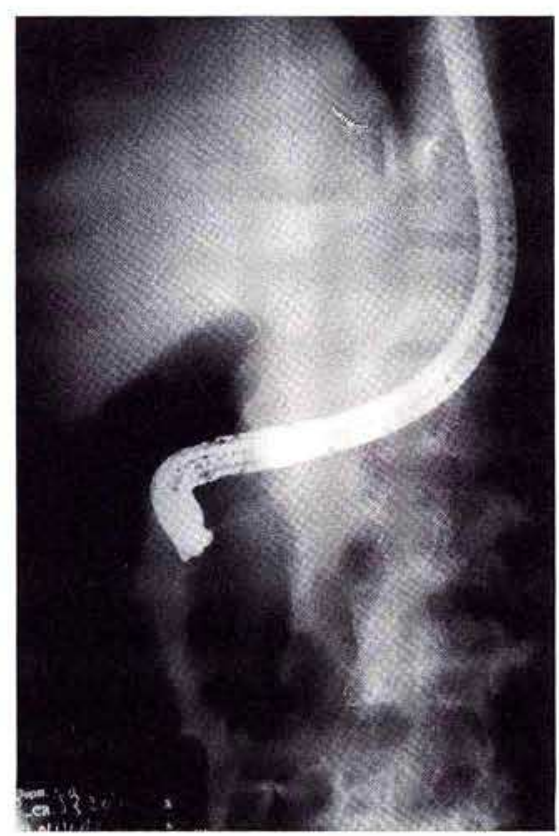


analysis $(3,4)$. Using our method, contrastfree bile can be collected even with a conventional ERCP catheter.

D. K. Bhasin, B. C. Sharma, S. Dhavan, K. Singh Department of Gastroenterology, Postgraduate Institute of Medical Education and Research, Chandigarh 160012, India

\section{References}

1. Leung JWC, Venezuela RR. Cholangiosepsis: endoscopic drainage and antibiotic therapy. Endoscopy 1991; 23: $220-3$.

2. Leung JWC, Chung SCS, Sung JJY, Banez VP, Li AKC. Urgent endoscopic drainage for acute suppurative cholangitis. Lancet 1989; i: 1307-9.

3. Schutz SM, Fitz JG, Brach MS, Baillie J. A new way to obtain contrast free bile during ERCP. Gastrointest Endosc 1996; 43: 231-2.
4. Misra SP, Dwivedi M. Obtaining contrast-free bile during ERCP. Gastrointest Endosc 1997; 45: 110.

Corresponding Author

Dr. D. K. Bhasin

1037, Sector $24 \mathrm{~B}$

Chandigarh 160023

India

Fax: $+91-172-543078$ 